\title{
ON PERFORMANCE EVALUATION AND PROTOCOLS DESIGN OF TREE PROTOCOL WITH COLLISION DETECTION
}

\author{
Jau-Hsiung Huang and Chia-Ning Wu \\ Department of Computer Science and Information Engineering \\ National Taiwan University \\ Taipei, Taiwan, R.O.C.
}

\begin{abstract}
Tree protocol is a simple collision resolution technique that both maintains stability without any complex estimation procedures and increases the achievable throughput. In this paper, we introduce tree protocol with collision detection which not only significantly improves the throughput and delay performance of the original protocol without collision detection, but also obtains a higher throughput and lower delay than Ethernet. In addition, when the channel propagation delay is small, we use a modified M/D/ 1 model to approximate the mean and distribution of the delay performance, and shows that such an approximation is in close agreement with the simulation results. Since M/D/1 achieves the lowest delay for all protocols, the delay performance of this protocol is very close to the best delay performance for all random access protocols when the channel propagation delay is small. Lastly, two modified protocols are given to resolve the synchronization problems in implementation.
\end{abstract}

\section{Introduction}

This paper considers the time-domain multiple accessing of a broadcast channel by a set of independent sources. In such a system, transmission collision is an inevitable problem. In order for a system to have a high percentage of successful transmissions, the behavior of users must be governed by a contention resolution Algorithm(CRA). There are many CRAs have been proposed [1,3,4,8-11,16-17]. Tree protocol, one of these CRAs, was first proposed by Capetanakis [8][9] and is the focus of this paper.

The idea of tree protocol is simple. When a collision occurs, say in the $k^{\text {th }}$ slot, all nodes involved in the collision will split into two subsets. The first subset transmits in slot $k+1$, and if the transmission in that slot is idle or successful, the second subset transmits in slot $k+2$. Alternatively, if another collision occurs in slot $k+1$, the first of the two subsets will split again and repeat the process. The second subset then just waits until the resolution of that collision in the first subset. The maximal throughput achievable with the original tree protocol is 0.43 [8].

Based on the original tree protocol, we include the idea of collision detection in the protocol as the celebrated CSMA/CD [7][15] to obtain a better throughput and delay performance. That is, whenever a collision is detected, all stations involved in this collision immediately stop transmitting and split into two subsets and transmit the first subset in the next slot. We will denote this tree protocol with collision detection as Tree/CD for the rest of this paper.

In this paper, we present the delay and throughput analysis of Tree/CD. There are some other papers work on the delay analysis of a CRA $[5,16]$; however, they derive only the upper bound and lower bound of the average delay by solving a series of complicated integral equations. Instead of that, we give a modified $M / D / 1$ model to approximate the mean transmission delay which is very simple and precise for many practical cases. Furthermore, the distribution of the system delay is also derived using the modified $M / D / 1$ model which is also verified through the simulation results. We show that the performance of our protocol is very close to the theoretical optimal with Poisson arrivals and deterministic transmission time when the channel propagation delay is small.

For a multi-accessing algorithm, the most difficult problem in implementation is to synchronize all stations at all time. In order to solve this problem, we propose two protocols which do not require strong synchronization as the original protocol. It is shown that the mean and variance of the system delay for both protocols are very close to the original protocol.

6C.3.1. 


\section{Model description of Tree/CD}

Our model is based on following assumptions:

(1) Slotted System: The channel time is partitioned into slots of length $\beta$, where $\beta$ is the maximal one-way source-destination propagation delay in a normalized basis. The normalization is performed such that the transmission time of a packet is unity.

(2) Infinite Set of Nodes.

(3) Poisson Arrival.

(4) Idle, Success, and Collision Immediately Feedback.

(5) Retransmission of Collisions.

Before introducing the Tree/CD protocol, we give some definitions at first. we define an enable allocation interval (EAI) as a time interval that packets arriving during which will be transmitted in the next slot. With reference to Fig. 1, the intervai $\left(\mathrm{s}_{i}, \mathrm{~s}_{i+1}\right]$ is called the $i^{\text {th }}$ enabled allocation interval, and its duration is denoted by $\alpha_{i}$. We then define a collision resolution period as the time needed to transmit all the packets arriving during the EAI. As shown in Fig. 1, the interval $\left(\mathrm{CT}_{i}, \mathrm{CT}_{i+1}\right]$ is called the $i^{\text {th }}$ collision resolution period (CRP), and its length is denoted by $\mathrm{T}_{i}$. It is nature to give EAI a preset size so that the system can achieve its maximal throughput. Suppose this preset size is $\alpha_{0}$, then the length of the $i^{\text {th }}$ EAI can be determined according to the formula

$$
\alpha_{i}=\min \left\{\mathrm{CT}_{i}-\mathrm{S}_{i}, \alpha_{0}\right\}
$$

where $\mathrm{CT}_{i}$ means the current time, and $\mathrm{S}_{i}$ means the starting point of the $i^{\text {th }}$ EAI. That is, $\alpha_{i}$ equals $\alpha_{0}$ unless that would extend the $\mathrm{i}^{\text {th }} \mathrm{EAI}$ beyond the present moment.

Finally, we use a variable $\sigma_{i}$ to indicate we are processing the left subinterval or the right subinterval, i.e.

$\sigma_{i}:\left\{\begin{array}{l}\mathrm{L} \text { if the allocation interval is at the left half } \\ \mathrm{R} \text { if the allocation interval is at the right half }\end{array}\right.$

Now, we present how the Tree/CD works. Suppose current EAI is located from $S_{i}$ to $S_{i}+\alpha_{i}$, when an idle occurs [Fig. 2 (a)], all stations will spend $\beta$ time to detect this idle. Then EAI advances for an interval $\alpha_{i}$; That is, $S_{i+l}=S_{i}+\alpha_{i}$. When a successful transmission occurs [Fig. 2 (b)], it takes the system one time unit to transmit this packet. The starting point of current EAI then proceeds $\alpha_{i}$, i.e. $S_{i+1}=S_{i}+\alpha_{i}$. In the cases of idle and success, $\alpha_{i+1}$ is reset to be $\min$ (CT$\left.\mathrm{s}_{i+1}, \alpha_{0}\right\}$.

A more complicated situation happens when there is a

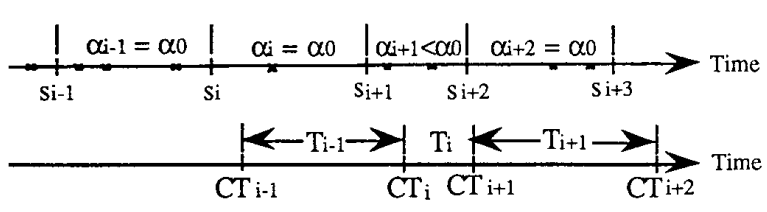

Fig. 1 Illustration of EAI and CRP.

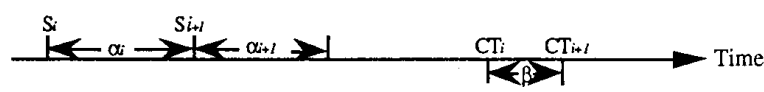

(a)
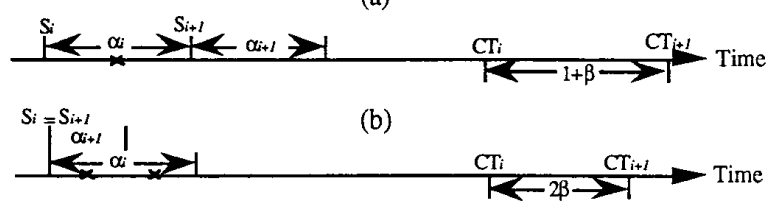

(c)

Fig. 2 Tree/CD protocol. (a) No arrival in EAI. (b) One arrival in EAI. (c) Two arrivals in EAI.

collision [Fig. 2 (c)]. The transmitters takes one $\beta$ to detect a collision and to cease the transmission, and another $\beta$ to clear the channel. The EAI is then split into two equal subintervals, and specifies the left subinterval as the EAI of the next slot $\left(\sigma_{i+1}=L\right)$. Thus, the starting point of current EAI is unchanged with $\alpha_{i+1}=\alpha_{i} / 2$. Alternatively, if another collision occurs in the next slot, then $L$ splits again (into $L L, L R$ ), and the second subset $(R)$ waits for the resolution of that collision.

\section{Throughput analysis of Tree/CD}

We evaluate the maximal stable throughput via

$$
\lambda_{\max }=\sup _{g} \frac{E\{\text { packets } / C R P\}}{E\{\text { time } / C R P\}}
$$

where $\mathrm{g}=\lim _{i \rightarrow \infty} \mathrm{g}_{i}$, and $\mathrm{g}_{i}$ is the Poisson intensity of the $i$ th EAI.

We evaluate $E\{$ packets/CRP $\}$ and $E\{$ time/CRP $\}$ as follows. Slightly modifying the tools used in [2], we get a Markov chain as shown in Fig. 3. The first term of a state ( $L$ or R) is defined as the status of the EAI which is in process, and the second term $(i)$ is defined as the number of splits that EAI has occurred.

Define $\mathrm{G}_{i}$ as the expected number of packets, a priori, in an interval that has been split $i$ times, then

\section{C.3.2.}




$$
\mathrm{G}_{i}=2^{-i} \lambda \alpha_{0}
$$

We give some notations as follows:

$\mathrm{P}_{\mathrm{RR}}(i)$ : the transition probability from node $(R, i)$ to $(R, 0)$. $\mathrm{P}_{R L}(i)$ : the transition probability from node $(R, i)$ to $(L, i+1)$. $\mathrm{P}_{L R}(i)$ : the transition probability from node $(L, i)$ to $(R, i)$.

$\mathrm{P}_{I}(i)$ : the probability with which node $(L, i)$ has an idle slot and goes into node $(L, i+1)$.

$\mathrm{P}_{C}(i)$ : the probability with which node $(L, i)$ has a collision and goes into node $(L, i+1)$.

It is easy to observe that

$$
\begin{aligned}
& \mathrm{P}_{R R}(0)=\left(1+\mathrm{G}_{0}\right) \mathrm{e}^{-\mathrm{G}_{0}} \\
& \mathrm{P}_{R L}(0)=1-\left(1+\mathrm{G}_{0}\right) \mathrm{e}^{-\mathrm{G}_{0}}
\end{aligned}
$$

and for $i>0$

$$
\begin{aligned}
& P_{L R}(i)=\frac{G_{i} e^{-G_{i}}\left(1-\mathrm{e}^{-G_{i}}\right)}{1-\left(1+G_{i-1}\right) \mathrm{e}^{-G_{i-1}}} \\
& \mathrm{P}_{R R}(i)=\frac{\mathrm{G}_{i} \mathrm{e}^{-\mathrm{G}_{i}}}{1-\mathrm{e}^{-\mathrm{G}_{i}}} \\
& \mathrm{P}_{R L}(i)=1-\frac{\mathrm{G}_{i} \mathrm{e}^{-\mathrm{G}_{i}}}{1-\mathrm{e}^{-\mathrm{G}_{i}}} \\
& \mathrm{P}_{I}(i)=\frac{\mathrm{e}^{-\mathrm{G}_{i}\left(1-\left(1+\mathrm{G}_{i}\right) \mathrm{e}^{-\mathrm{G}_{i}}\right)}}{1-\left(1+\mathrm{G}_{i-1}\right) \mathrm{e}^{-\mathrm{G}_{i-1}}} \\
& \mathrm{P}_{C}(i)=\frac{1-\left(1+\mathrm{G}_{i}\right) \mathrm{e}^{-\mathrm{G}_{i}}}{1-\left(1+\mathrm{G}_{i-1}\right) \mathrm{e}^{-\mathrm{G}_{i-1}}}
\end{aligned}
$$

The analysis of this chain is simple since no state can be entered more than once before returning to $(R, 0)$. Let $S_{L^{(i)}}$ and $S_{R}(i)$ denote the probability of entering into state $(L, i)$ and $(R, i)$ before returning to $(R, 0)$ respectively. Then

$$
\begin{aligned}
& \mathrm{S}_{L}(1)=1-\mathrm{P}_{R R}(0) \\
& \mathrm{S}_{R}(i)=\mathrm{S}_{L}(i) \cdot \mathrm{P}_{L R}(i) \\
& \mathrm{S}_{L}(i+1)=\left(1-\mathrm{P}_{L R}(i)\right) \cdot \mathrm{S}_{L}(i)+\left(1-\mathrm{P}_{R R}(i)\right) \cdot \mathrm{S}_{R}(i)
\end{aligned}
$$

And thus we have

$$
\begin{aligned}
& \mathrm{E}\{\text { time } / \mathrm{CRP}\}=\beta \mathrm{e}^{-\mathrm{G}_{0}}+(1+\beta) \mathrm{G}_{0} \mathrm{e}^{-\mathrm{G}_{0}} \\
& +\sum_{i=1}^{\infty} \mathrm{S}_{L}(i)\left(\mathrm{P}_{C}(i) \cdot 2 \beta+\mathrm{P}_{I}(i) \cdot \beta\right)+\sum_{i=1}^{\infty} \mathrm{S}_{L}(i) \mathrm{P}_{L R}(i) \cdot(1+\beta) \\
& +\sum_{i=1}^{\infty} \mathrm{S}_{R}(i) \cdot \mathrm{P}_{R L}(i) \cdot 2 \beta+\sum_{i=1}^{\infty} \mathrm{S}_{R}(i) \cdot \mathrm{P}_{R R}(i) \cdot(1+\beta)
\end{aligned}
$$

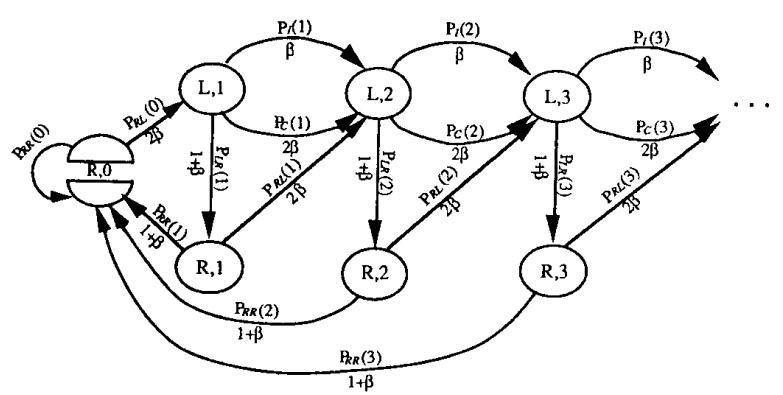

Fig. 3 The Markov chain of Tree/CD.

Now we evaluate the average number of packets processed in a CRP. For the initial size $\alpha_{0}$, this quantity should be $\lambda \alpha_{0}$. However, if left intervals have collisions, then the corresponding right-hand intervals are returned to the waiting interval and the length of the CRP is less than $\alpha_{0}$. Denote $f$ as the fraction of $\alpha_{0}$ returned in this way during a CRP, then $\lambda \alpha_{0}(1-f)$ is the average number of packets processed in a CRP. The expected value of $f$ can be expressed as

$$
\mathrm{E}[f]=\sum_{i=1}^{\infty} S_{L}(i) \cdot \mathrm{P}_{C}(i) \cdot 2^{-i}
$$

And the expected number of packets transmitted in a CRP is

$$
\mathrm{E}(\text { packets } / \mathrm{CRP}\}=\lambda \alpha_{0}(1-\mathrm{E}\{f\})
$$

Applying equations (12) and (14) into (1), we can evaluate the maximal throughput of Tree/CD. The maximal throughput under different values of $\beta$ is illustrated in Table 1 .

\begin{tabular}{cc}
\hline \hline$\beta$ & $\lambda_{\max }$ \\
\hline 0.01 & 0.975432 \\
0.05 & 0.885212 \\
0.1 & 0.793491 \\
0.5 & 0.433861 \\
\hline
\end{tabular}

Table 1. The maximal throughput of the improved Tree/CD under some values of $\beta$.

\section{C.3.3.}




\section{Delay analysis of Tree/CD}

For any contention resolution algorithm, delay analysis is always a challenging problem, because the exact distribution of the waiting time of a packet is extremely difficult to find. In this paper, we try to use an M/D/1 model to approximate the average transmission delay of Tree/CD. We observe that the service time of Tree/CD is roughly fixed. A packet takes $1+\beta$ time unit for a successful transmission and an extra overhead when a collision is suffered. If $\beta$ is small, the overhead is negligible. Therefore the service time of Tree/CD is roughly fixed to be $1+\beta$, and the behavior of Tree/CD is somewhat like an $M / D / 1$ queue. By approximating Tree/CD with an $M / D / 1$ queue, we can easily write down the distribution of delay of Tree/CD if only we can find the mean service time of it.

The mean service time of a packet is calculated as follows. If a packet arrives during an EAI, it is obvious that there must be at least one packet in that EAI. Suppose $\mathrm{p}_{+}(n)$ is the probability of an $n$-arrival EAI "seen" by a packet (Assume the total number of arrivals, including the packet itself, is equal to $n$.), and $N$ is the number of packets comes during an EAI of length $\alpha=\min \left(\mathrm{CT}-\mathrm{S}, \alpha_{0}\right)$, then $\mathrm{p}_{+}(n)$ can be written as

$$
\mathrm{p}_{+}(n)=\operatorname{Prob}[N=n \mid n>0]=\frac{\mathrm{e}^{-\lambda \alpha}(\lambda \alpha)^{n}}{n !\left(1-\mathrm{e}^{-\lambda \alpha}\right)}
$$

Suppose $\mathrm{T}_{n}$ to be the expected time of a CRP which starts with $n$ packets, and $\mathrm{K}_{n}$ to be the expected number of packets transmitted successfully in a CRP which begins with $n$ packets, the mean service time $\bar{X}$ can be approximated as

$$
\overline{\mathrm{X}}=\frac{\sum_{n=1}^{\infty} \mathrm{p}_{+}(n) \mathrm{T}_{n}}{\sum_{n=1}^{\infty} \mathrm{p}_{+}(n) \mathrm{K}_{n}}
$$

We derive $\mathrm{T}_{n}$ and $\mathrm{K}_{n}$ in the APPENDIX

Now we can apply some formulas of $M / D / 1$ to the Tree/CD system. The Laplace transform of the distribution of total time spent in an $\mathrm{M} / \mathrm{D} / 1$ system can be expressed as [12]

$$
s^{*}(s)=(1-\rho) \frac{s e^{-X s}}{s-\lambda+\lambda e^{-X s}}
$$

This is the Laplace transform of the distribution of delay of Tree/CD. Hence, the mean delay of Tree/CD can be found by computing its first moment

$$
\mathrm{T}=\frac{\mathrm{d} \mathrm{S}^{*}(\mathrm{~s})}{\mathrm{ds}} \mathrm{I}_{\mathrm{S}=0}=\overline{\mathrm{X}}+\frac{\rho \overline{\mathrm{X}}}{2(1-\rho)}
$$

where $\rho=\lambda \bar{X}$.

Substituting $\overline{\mathrm{X}}$ from (16) into (20), the mean delay of Tree/CD is obtained. Given $\beta=0.01$, and 0.05 , the theoretical average delay $\mathrm{T}$ under different $\lambda$ and the results of simulations (based on $2.5 \times 10^{5}$ time unit per simulation point) is illustrated in Fig. 4. It shows that our approximations are very close to the simulation results.

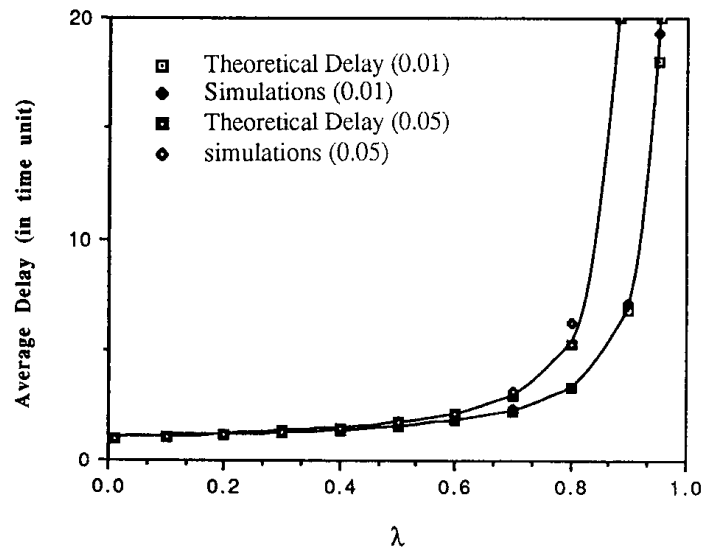

Fig. 4. Expected delay of $M / D / 1$ model versus simulation results.

(Number in the parenthesis is the value of $\beta$.)

In order to confirm the validity of (19), we compute the variance of delay as

$$
\operatorname{Var}=\frac{\rho \bar{X}^{2}(4-\rho)}{12(1-\rho)^{2}}
$$

The curve of the variance of delay under different $\beta$ is plotted as Fig. 5. When $\beta$ is moderately small, the simulation results are also very close to the theoretical variance of delay. This provides an further evidence for the analysis we have done in this section.

\section{C.3.4.}




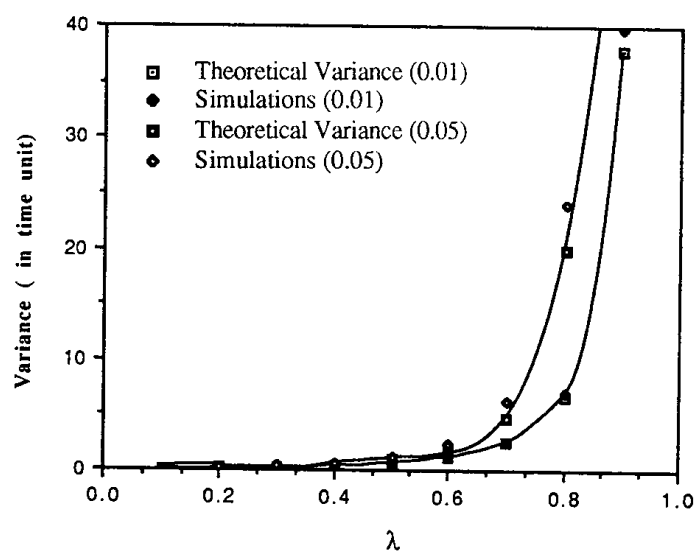

Fig. 5. Expected variance of the $M / D / 1$ model versus simulation results.

(Number in the parenthesis is the value of $\beta$.)

\section{Comparison between Tree/CD and Ethernet}

Ethernet is the most popular commercial product today, so we choose it as a standard to compare with Tree/CD. We make this comparison in three respects: throughput, delay, and variance.

\section{(1) Throughput}

The maximal stable throughput of Ethernet can be obtained in [2] and will not be repeated here. The maximal stable throughputs of Ethernet under some values of $\beta$ are illustrated in Table 2. By comparing Table 2 with Table 1 , we can see that the throughput of Tree/CD is higher than Ethernet. However, the most significant advantage of Tree/CD over Ethernet is on the mean and variance of the system delay as will be shown later.

\section{(2) Delay}

We offer some simulation results to show that Tree/CD is also superior to Ethernet in transmission delay. Given $\beta=0.01,0.05$, and 0.1 , the simulated delays of Ethernet and Tree/CD is illustrated in Fig. 6 These results are obtained on a $2.5 \times 10^{5}$ time unit basis. It is shown that at light load and medium load, the delay performances of Tree/CD and Ethernet are very close to each other. However, when the load of the system is heavy, the delay performance of Tree/CD is clearly better than that of Ethernet. It is obvious especially when $\beta$ increases.

\begin{tabular}{cl}
\hline \hline$\beta$ & $\lambda_{\max }$ \\
\hline 0.01 & 0.967950 \\
0.05 & 0.857961 \\
0.1 & 0.751254 \\
0.5 & 0.376571 \\
\hline
\end{tabular}

Table 2. The maximal throughput of Ethernet under some values of $\beta$.

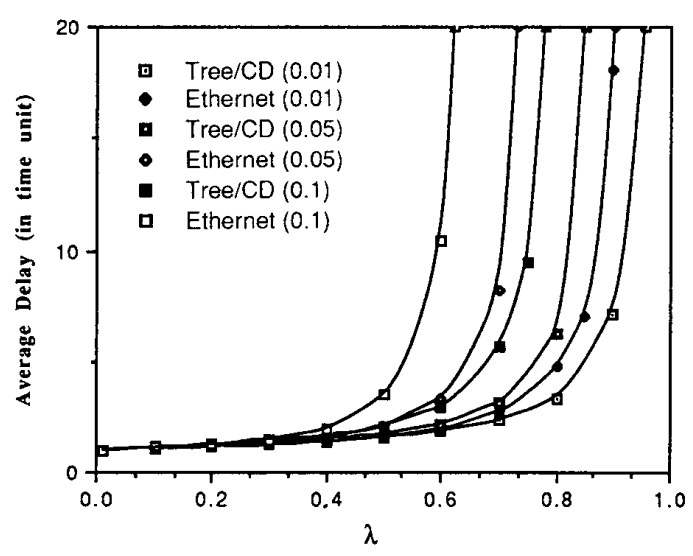

Fig.6. The average delay of Tree/CD and Ethernet.

(Number in the parentheses is the value of $\beta$.)

\section{(3) Variance}

As mentioned before, Ethernet adopt a retransmission mechanism called Truncated Binary Backoff algorithm. In this algorithm, if a packet is transmitted first time, it will be transmitted in the immediate slot; however, if a packet has been transmitted $n$ times before, it will be transmitted in the next $2^{n}$ slots $(0 \leq n \leq 10)$ with equal probability. Therefore, an "older" packet is tend to be transmitted with a longer delay than a "younger" one in Ethernet. This gives Ethernet an inherent great variance.

On the contrary, in the case of Tree/CD, packets are served in a way of first-come-first-serve and therefore guarantees a reasonable variance. To some applications, such as the real time system, the low variance characteristic is considered more important than the low delay. As shown in Fig. 7, it is undoubted that Tree/CD is more suitable for real-time processing than Ethernet.

\section{C.3.5.}




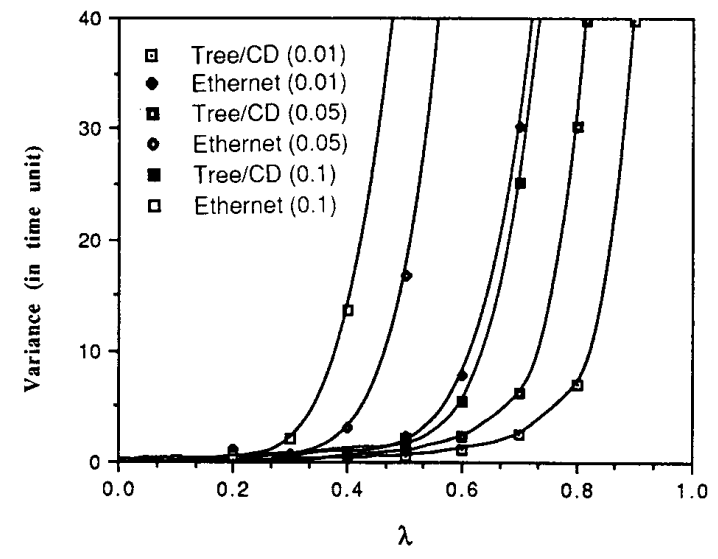

Fig. 7. The variance of Tree/CD and Ethernet.

(Number in the parentheses is the value of $\beta$.)

\section{Two protocols for implementations: TA and MFA}

Though Tree/CD has many good characteristics which have been introduced previously, it is subject to some problems. One of these problems is that Tree/CD requires continuous channel sensing by all users to ascertain the exact location of current EAI. The second problem with Tree/CD is the synchronization problem occurs when a new user joins the system. Besides, Tree protocol is not robust to feedback errors; if the feedback from an idle slot is mistaken as a collision, EAI will split infinitely.

Among these problems, robustness to feedback errors can be easily achieved by specifying a maximal number $h$ of successive idles after a collision. On the $h^{\text {th }}$ successive idle after a collision, system will not split current EAI but just transmit the right interval. However, the other two problems are somewhat more difficult; thus we propose two protocols to implement Tree/CD in order to solve these problems.

(1) Toss-Up Algorithm (TA)

The reason for a station to keep continuous channel sensing is to know the location of EAI, so that the station can tell if it is its turn to transmit. If we have another way to specify the transmitter, we can effectively avoid problems mentioned above. In TA, when a collision occurs, each station owning a collided packet will toss a coin. If a head shows up, the station is in the left subset and has the right to transmit in the next slot; if a tail shows up, the station is in the right subset and must wait. Such a modification eliminates the use of EAI, and thus rids a station of the need to monitor the channel. However, by allowing stations not to continuously monitor the channel state, a newly arriving packet needs a way to tell when the current CRP will end. To accomplish this, we add a one-bit field $\mathrm{R}$ in the packet format as shown in Fig. 8.

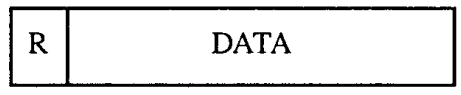

Fig. 8. Packet format of TA.

When a station located in the right interval transmits a packet, it sets the $R$-bit to be 1 ; otherwise, $R=0$. A new packet experiencing no collision always has $\mathrm{R}=1$. The reason that we add this bit is to solve the problem of synchronization whenever a new user enters the system. An interesting property of Tree/CD is that a CRP always ends with a successful transmission with $\mathrm{R}=1$. Therefore, a station learns that a CRP is over by hearing a successful transmitted packet with $\mathrm{R}=1$. Some transmission examples are presented in Fig.9.

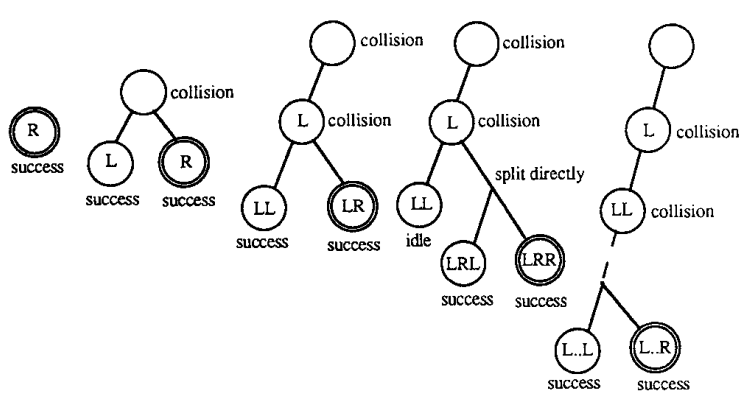

Fig. 9. Five different cases of splitting.

The flowchart of TA is shown in Fig. 10. The major advantage of TA is that this protocol eliminates the need for all stations to continuously monitor the channel. A station senses the channel only when it has packets to transmit. When the transmission is over, the station no longer needs to sense the channel. TA also solves the problem of allowing new stations to join the system. More importantly, since all stations do not use packet arrival time to split the tree and they do not have to keep track of when the CRP will end, this protocol avoids the synchronization problem, which is the most serious problem of the original Tree/CD protocol.

\section{(2) Multifield Frame Algorithm (MFA)}

TA has many good characteristics: simplicity, effectivity,

\section{C.3.6.}




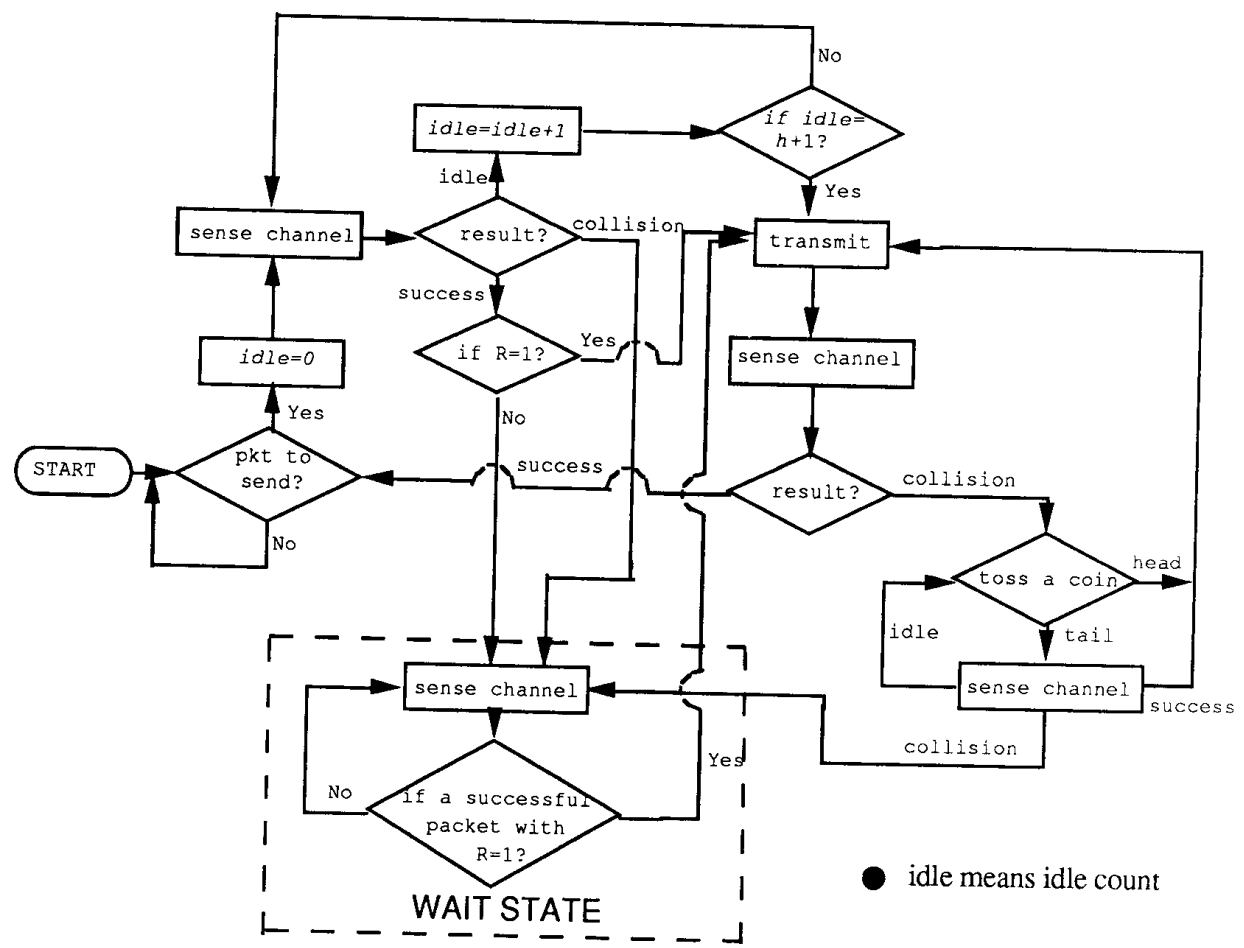

Fig. 10. The flowchart of Toss-Up Algorithm.

distributed control, and no synchronization problem. But as it does not transmit packets in a first-come-first-serve way, it inevitably suffers the same problem of "higher variance" as Ethernet. Therefore, we propose another alternative protocol: Multifield Frame Algorithm. MFA employs a special packet format as shown in Fig. 11.

\begin{tabular}{|l|l|l|l|}
\hline R & EEAI & CT & \\
\hline
\end{tabular}

EEAI: Ending Time of EAI

CT: Current Time

Fig. 11. Packet format of MFA.

The effect of the R-bit in this algorithm is the same as that in TA. The additional EEAI and CT fields are used to record the ending time of EAI and the current time respectively. Right before a packet is sent, the station copies the current time in its clock into the CT field in the packet. That is, CT contains the current time when the station begins transmitting. Meanwhile, the station computes the ending time of EAI by assuming itself as the last successfully transmitted packet in the EAI. With these fields, MFA holds a behavior like Tree/CD; but a station needs to sense the channel only when it has packets to send.

We describe the operation of MFA protocol below. Like $\mathrm{TA}$, when a station has packets to send, it must keep listening to the channel until a successfully transmitted packet with $R=1$ is heard. Such a packet means that the current CRP is over, and a new CRP is going to start immediately. The stations which have packets to send can obtain all necessary information from this packet, such as the current time (for synchronization use) and the new EAI interval to be resolved by the next CRP. (We assume that the preset maximal size of EAI, or $\alpha_{0}$, is known by all stations.) The stations first reset their clock according to the CT field of that packet, and then copy the value of EEAI to variable $S$, which means the starting time of this new EAI. (The ending point of an EAI is the starting point of the next EAI.) Now that the starting point and the length of EAI is found, the station can then check whether its packet arrived during the interval from $S$ to $\min \left(S+\alpha_{0}, C T\right)$.

If does, the station sets the $\mathrm{R}$ field of its packet to be $1, \mathrm{CT}$ field to be the current time, EEAI field to be $\min \left(S+\alpha_{0}, C T\right)$, and sends the packet. However, if its packet's arrival time is 
not within EAI, the station will just wait until the next successfully transmitted packet with $\mathrm{R}=1$ shows up. After having transmitted such a packet, the station keeps listening to the channel. If the feedback is not successful, the EAI of that station will split, and the station retransmits its packet according to EAI. Now the EEAI field of the retransmitted packet is no longer $\min \left(S+\alpha_{0}, C T\right)$, but $S+\alpha$, where $S$ and $\alpha$ is changeable according to the algorithm listed in section II.

A problem may occur when a station enters an idle system Because there is no successful packet sensed, the station may puzzle over how to set the EEAI field. Our solution is that when a station hears $h+1$ successive idles, which means that the system is idle, it directly sets R to be 1 , EEAI field to be the packet's arrival time, CT field to be its clock time, and then transmits the packet immediately.

Now we discuss on the performance of TA and MFA. Both TA and MFA use some extra bits to keep the information, and thus will affect their throughputs. In TA, the overhead is only one bit per packet; hence, the degradation in throughput can be neglected. In MFA, although the overhead contains three fields, the degradation in throughput is still negligible if the data field is long enough. Suppose the length of EEAI, CT, and DATA field is $r, s$, and $d$ bits respectively, the maximal throughput will degrade to be $\frac{d}{d+r+s+1} \lambda_{\max }$ ,where $\lambda_{\max }$ is the achievable maximal throughput of Tree/CD. For most systems, DATA field is much longer than EEAI field and CT field, i.e., $d \gg r$ and $d \gg>$. Therefore, the effect of the overhead on throughput in MFA is minor.

As to the mean delay of TA and MFA, Fig. 12 shows the average delay of TA, MFA, Tree/CD, and Ethernet for $h=5$. It is obvious that Tree/CD owns the minimal delay. When $\lambda$ is greater then a threshold (e.g. about 0.6 for $\beta=0.01$ and 0.5 for $\beta=0.05$ and 0.1 ), the average delays of TA and MFA are smaller than that of Ethernet.

As to the mean variance of delay, Fig. 13 shows the average variance of delay of TA, MFA, Tree/CD, and Ethernet. Since Tree/CD and MFA transmit packets in a FCFS way, they both own the least variance. For TA and Ethernet, when $\lambda$ is small, the variance of TA is slightly greater than that of Ethernet; when $\lambda$ is moderately large, however, TA owns a better variance property than Ethernet.

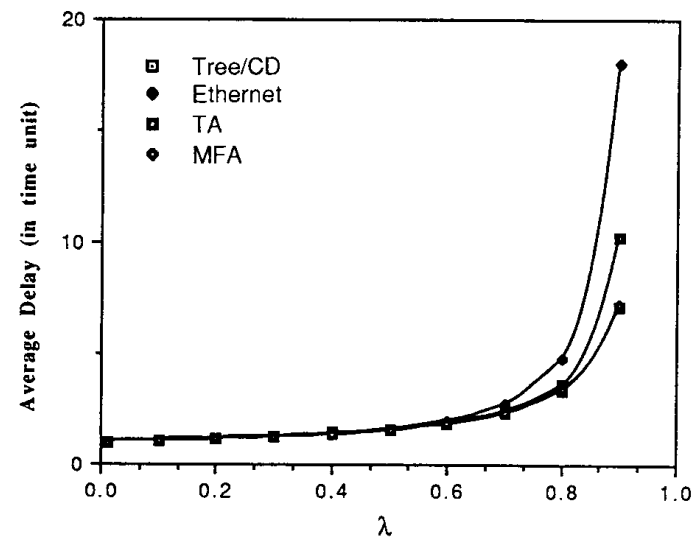

Fig. 12. The average delays of Tree/CD, Ethernet, TA and MFA when $\beta=0.01$.

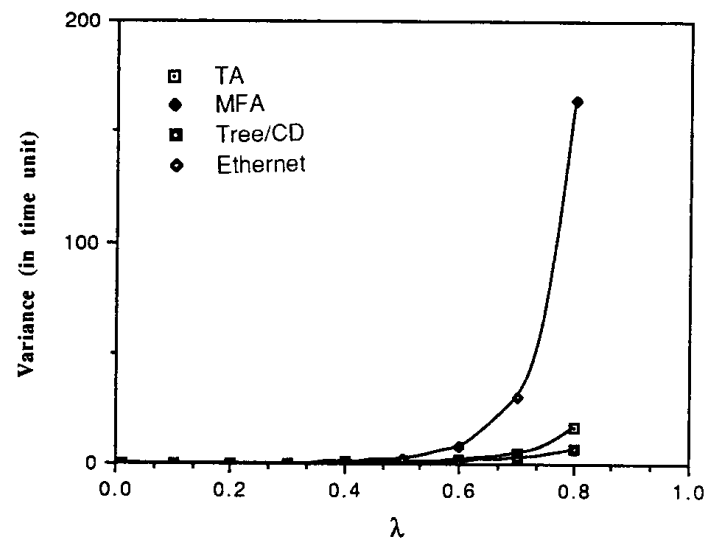

Fig. 13. The variance of Tree/CD, Ethernet, TA and MFA when $\beta=0.01$.

\section{Conclusion}

In this paper we present Tree/CD protocol, an enhanced version of the Tree protocol by employing the idea of collision detection. We show that the performance (throughput, delay, and variance) of the original Tree protocol is greatly improved by giving stations the capability to detect and abort an unsuccessful transmission immediately. Besides the characteristic of high throughput, Tree/CD also owns lower delay and variance, which are important requirements of a realtime system.

6C.3.8. 
After demonstrating some attractive characteristics of Tree/CD, we give a detail analysis of the system time of the protocol. When $\beta$ is small in comparison with the packet transmission time, we show that our analytical results are very precise by simulations.

Finally, we proposed TA and MFA to resolve the synchronization problem while retaining the good properties which Tree/CD has. As our simulation results show, the performance of these two protocols are very close to the Tree/CD protocol; hence, they prove themselves as good candidates in implementation. Further, since these protocols have very small variance in delay, they are also good candidates to support real-time traffic.

\section{References}

[1] A. G. Greenberg, P. Flajokt, and R. E. Ladner , "Estimating the multiplicity of conflicts to speed their resolution in multiple access channels," J. ACM, vol. 34, April 1987.

[2] Bertsekas, and Gallager, Data networks, Prentice-Hall International, Inc.,1987.

[3] D. Towsley, and G. Venkatesh, "Window random access protocols for local computer networks," IEEE Trans. Comp., vol. C-31, Aug. 1982.

[4] E. Gulko, "Tree-based multiaccess protocol where collision multiplicity are known, "IEEE Trans. Commun., vol. COM-33, Sep., 1985.

[5] J. C. Huang, and T. Berger, "Delay analysis of intervalsearching contention resolution algorithm," IEEE Trans. Inform. Theory, vol. IT-31, NO. 2, Mar. 1985.

[6] J. C. Huang, and T. Berger, "Delay analysis of 0.487 contention resolution algorithms," IEEE Trans. Commun., vol. COM-34, NO. 9, Sep. 1986.

[7] J. F. Schoch, Y. K. Dalal, and D. D. Redell, " Evolution of the Ethernet local computer network," IEEE Comp., vol. 15, Aug. 1982.

[8] J. I. Capetanakis, "The multiple access broadcast channel: protocol and capacity considerations," IEEE Trans. Inform. Theory, vol. IT-25, Sep. 1979.

[9] J. I. Capetanakis, "Generalized TDMA : the multiaccessing tree protocol," IEEE Trans. Commun., vol. COM-27, Oct. 1979.

[10] J. L. Massey, "Collision-resolution algorithms and random access communications," (Report UCLA-ENG8016). Los Angeles: University of California, 1980.
[11] J. Mosely, and P. A. Humblet, "A class of efficient contention resolution protocol for multiple access channels," IEEE Trans. Commun., COM-33, 1985.

[12] L. Kleinrock, Queueing system, Vol. I: Theory, New York: John Wiley \& Sons., 1975.

[13] N. Pippenger, "Bounds on the performance of protocols for a multiple access broadcast channel," IEEE Trans. Inform. Theory, IT-27, 1981.

[14] P. A. Humblet, "On the throughput of channel access algorithms with limited sensing," IEEE Trans. Commun. COM-34, 1986.

[15] R. M. Metcalf, and D. R. Boggs, "Ethernet: distributed packet switching for local computer networks," Commun ACN. 1976.

[16] T. Berger, and J. C. Huang, "A conflict resolution algorithm with multibit feedback," IEEE Trans. Inform. Theory, Dec. 1983.

[17] T. Berger, N. Mehravari, D. Towsley, and J. K. Wolf, "Random multiple-access communication and group testing," IEEE Trans. Commun., vol. COM-32, July 1984.

\section{APPENDIX: Computation of $T_{n}$ and $K_{n}$}

We work on $\mathrm{T}_{n}$ first. It is trivial to find $\mathrm{T}_{0}=\beta$ and $\mathrm{T}_{1}=1+\beta$. For $n \geq 2$, all packets involved in the collision will be split into two groups of size $i$ and $n-i$ with probability $\left(\begin{array}{l}n \\ i\end{array}\right)\left(\frac{1}{2}\right)^{n}$. If $i=0$, the right subgroup will be split directly before transmission, thus takes $\mathrm{T}_{0}+\mathrm{T}_{n}-2 \beta$ time units. If $i=1$, it takes $\mathrm{T}_{1}+\mathrm{T}_{n-1}$ time units. If $i \geq 2$, this means another collision is suffered, and Tree/CD will just forget the $n-i$ packets in the right side. $\mathrm{T}_{n}$ can thus be expressed as

$$
\begin{aligned}
\mathrm{T}_{n}=2 \beta & +\left(\mathrm{T}_{0}+\mathrm{T}_{n}-2 \beta\right)\left(\begin{array}{l}
n \\
0
\end{array}\right)\left(\frac{1}{2}\right)^{n}+\left(\mathrm{T}_{1}+\mathrm{T}_{n-1}\right)\left(\begin{array}{l}
n \\
1
\end{array}\right)\left(\frac{1}{2}\right)^{n} \\
& +\sum_{i=2}^{n} \mathrm{~T}_{i}\left(\begin{array}{l}
n \\
i
\end{array}\right)\left(\frac{1}{2}\right)^{n}
\end{aligned}
$$

And we obtain a general expression of $\mathrm{T}_{n}$

$$
\begin{aligned}
& \mathrm{T}_{0}=\beta \\
& \mathrm{T}_{1}=1+\beta
\end{aligned}
$$




$$
\mathrm{T}_{n}=\frac{2 \beta-\left(\frac{1}{2}\right)^{n} \beta+\mathrm{n}\left(\frac{1}{2}\right)^{n}(1+\beta)+n\left(\frac{1}{2}\right)^{n} \mathrm{~T}_{n-1}+\sum_{i=1}^{n-1}\left(\begin{array}{l}
n \\
i
\end{array}\right)\left(\frac{1}{2}\right)^{n} \mathrm{~T}_{i}}{1-\left(\frac{1}{2}\right)^{n-1}}
$$

for $n \geq 2$.

(25)

Then we work on $K_{n}$. It is also trivial to find $\mathrm{K}_{0}=0$ and $\mathrm{K}_{1}=1$. For $n \geq 2$, packets involved in the collision will be split into subgroup of the form $(0, n),(1, n-1)$ or $(i, n-i)$ where $i \geq 2$. In the case of $(0, n)$, the expected number of successfully transmitted packets is still $K_{n}$. In the case of $(1, n-1)$, one packet in the left half will be transmitted successfully, and the expected successful transmission in the right half is $\mathrm{K}_{n-1}$; thus, $\mathrm{K}_{n}=\mathrm{K}_{1}+\mathrm{K}_{n-1}$. In the case of $(i, n-i)$ with $i \geq 2$, as mentioned before, $n-i$ packets located in the right side will be just forgot; thus, $\mathrm{K}_{n}=\mathrm{K}_{i}$. Therefore, for $\mathrm{n} \geq 2, \mathrm{~K}_{n}$ is expressed as

$\mathrm{K}_{n}=\left(\begin{array}{l}n \\ 0\end{array}\right)\left(\frac{1}{2}\right)^{n} \mathrm{~K}_{0}+\left(\begin{array}{l}n \\ 1\end{array}\right)\left(\frac{1}{2}\right)^{n}\left(\mathrm{~K}_{1}+\mathrm{K}_{n-1}\right)+\sum_{i=2}^{n}\left(\begin{array}{l}n \\ i\end{array}\right)\left(\frac{1}{2}\right)^{n} \mathrm{~K}_{n}$

And we obtain a general expression of $\mathrm{K}_{\mathrm{n}}$

$$
\begin{aligned}
& \mathrm{K}_{0}=0 \\
& \mathrm{~K}_{1}=1
\end{aligned}
$$

$$
\mathrm{K}_{n}=\frac{n\left(\frac{1}{2}\right)^{n}+n\left(\frac{1}{2}\right)^{n} \mathrm{~K}_{n-1}+\sum_{i=2}^{n-1}\left(\begin{array}{l}
n \\
i
\end{array}\right)\left(\frac{1}{2}\right)^{n} \mathrm{~K}_{i}}{1-\left(\frac{1}{2}\right)^{n-1}} \text { for } n \geq 2
$$

6C.3.10. 